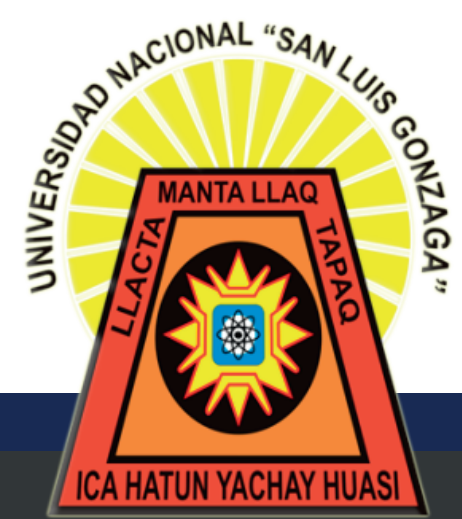

p-ISSN 2223-2893

e-ISSN 2225-6989

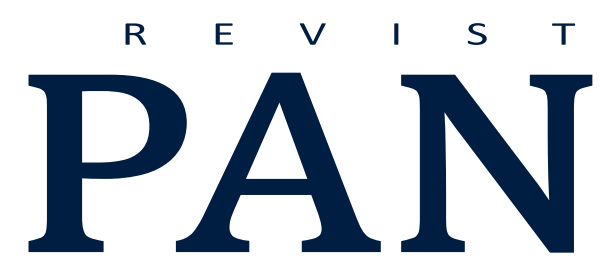

UNIVERSIDAD NACIONAL SAN LUIS GONZAGA. ICA, PERÚ
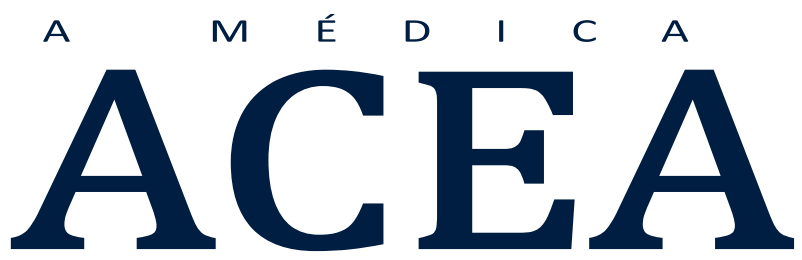

FACULTAD DE MEDICINA HUMANA "DANIEL ALCIDES CARRIÓN"

ARTÍCULO DE REVISIÓN:

VOLUMEN 10 NÚMERO 1

PUBLICACION CUATRIMESTRAL

ENERO - ABRIL

2021

REVISIÓN SISTEMÁTICA: CAMBIOS EN EL TAMAÑO DE

LA ZONA FOVEAL AVASCULAR EN PACIENTES CON DIAGNÓSTICO DE GLAUCOMA Y EN PACIENTES SANOS.

\title{
AUTORES:
}

CARLOS BARBA CHIRINO

JAZZMIN ARANDA CALIPUY

INDEXADA EN:

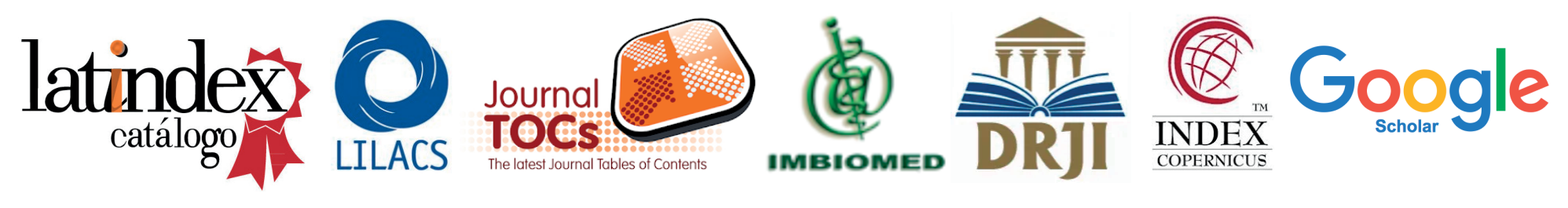

REVISTAS.UNICA.EDU.PE 


\title{
REVISIÓN SISTEMÁTICA: CAMBIOS EN EL TAMAÑO DE LA ZONA FOVEAL AVASCULAR EN PACIENTES CON DIAGNÓSTICO DE GLAUCOMA Y EN PACIENTES SANOS.
}

\author{
SYSTEMATIC REVIEW: CHANGES IN THE SIZE OF THE FOVEAL AVASCULAR ZONE IN PATIENTS \\ WITH A DIAGNOSIS OF GLAUCOMA AND IN HEALTHY PATIENTS.
}

Barba-Chirino Carlos', Aranda-Calipuy Jazzmin².

1. Instituto Regional de Oftalmología. Trujillo. Perú. Universidad Privada Antenor Orrego. Trujilllo. Perú. Médico Oftalmólogo.
2. Universidad Privada Antenor Orrego. Trujillo. Perú. Estudiante del XIII ciclo de Medicina Humana.

DOI: $10.35563 /$ rmp.v10i1.407

Correspondencia:

Aranda Calipuy Jazzmin

Eliana

Dirección: Mz "E" lote 7 Urb.

San José de California

Teléfono: 977379104

Contribuciones de autoría: $\mathrm{CBCH}$ : Diseño del estudio, análisis, redacción, revisión y aprobación final del manuscrito. búsqueda de literatura, análisis bibliométrico, corrección metodológica del manuscrito.

JAC: Procesamiento de datos, análisis de datos, redacción.

Conflicto de intereses: no existen conflictos de intereses del autor o autores de orden económico, institucional, laboral o personal.

Financiamiento:

Autofinanciado.

\section{Cómo citar:}

Barba-Chirino

Aranda-Calipuy J. Revisión sistemática: Cambios en el tamaño de la zona foveal avascular en pacientes con diagnóstico de glaucoma y en pacientes sanos. Rev méd panacea. 2020;10(1) 54-59. DOI:

10.35563/rmp.v10i1.407

\section{RESUMEN}

Se llevó a cabo un estudio para establecer si existen cambios del tamaño de la zona foveal avascular en pacientes con glaucoma a comparación de pacientes sanos; por medio de una Revisión Sistemática y Metaanálisis. Se incluyeron artículos originales que cumplan los criterios de inclusión y exclusión en los que se relacionen las variables de cambio en el tamaño de la zona foveal avascular en pacientes con glaucoma y pacientes sanos. En la revisión sistemática realizada se encontró que existen diferencias significativas respecto al tamaño de la zona foveal avascular entre pacientes con diagnóstico de glaucoma o pacientes sanos; las investigaciones incluidas en el presente metanalisis correspondieron todas al diseño transversal, con tamaños muestrales reducidos y con un nivel de sesgo mínimo solo en los parámetros de selección y resultado; ademas de las diferencias observadas respecto al tamaño de la zona foveal avascular entre pacientes con diagnóstico de glaucoma o pacientes sanos; también se identificaron como significativas las diferencias para otro parámetros como el perímetro y la circularidad.

Palabras clave: Oftalmopatía, Glaucoma, Zona foveal avascular.

\section{ABSTRACT}

A study was carried out to establish whether there are changes in the size of the avascular foveal zone in patients with glaucoma compared to healthy patients; through a Systematic Review and Meta-analysis. Original articles that met the inclusion and exclusion criteria were included in which the variables of change in the size of the avascular foveal zone are related in patients with glaucoma and healthy patients. In the systematic review carried out, it was found that there are significant differences regarding the size of the avascular foveal zone between patients with a diagnosis of glaucoma or healthy patients; The investigations included in this meta-analysis all corresponded to a cross-sectional design, with small sample sizes and with a minimal level of bias only in the selection and outcome parameters; in addition to the differences observed regarding the size of the avascular foveal zone between patients with a diagnosis of glaucoma or healthy patients; Differences for other parameters such as perimeter and circularity were also identified as significant.

Keywords: Glaucoma, foveal avascular zone, visual field. 


\section{INTRODUCCIÓN}

El glaucoma es considerado la segunda causa de ceguera a nivel mundial, se estima que para este año 2020 habría 79.6 millones de casos, de cual el Glaucoma de Angulo Abierto representará el $74 \% 1$. Es una neuropatía óptica en el cual hay una degeneración de las células ganglionares de la retina2. Es importante discernir de la hipertensión ocular, aunque influye y es una de las causas más importantes para el progreso de esta enfermedad, no es esencial3.

Se clasifica en glaucoma de ángulo abierto, el más frecuente. Se debe a un incremento en productividad del humor acuoso más una menor salida de este flujo, primario o secundario; Glaucoma de ángulo cerrado: se relaciona con el ángulo obstruido lo que imposibilita el drenaje de la cámara anterior, puede ser primario secundario; Glaucoma congénito, son los menos frecuentes; Glaucoma de tensión normal, entre otros4

Es necesario mencionar los diferentes factores de riesgo: edad, el más fundamental, mayores de 40 años es $2 \%$ y mayores de 70 años es $6 \% 5$. Presión intraocular, es más notable para su evolución de GAA y puede recibir un tratamiento. Su límite normal es $21 \mathrm{mmHg} 6$ Además la raza (africanos más frecuentes), historia familiar, factores oculares (miopía, hipermetropía), otros (uso de corticoides).

La zona foveal avascular es un área en el centro de la mácula que no tiene capilares 6 además es una zona especializada de la retina en la que se acerca a la región donde hay más fotorreceptores7. La ausencia de vasos más la intercalación del tejido retiniano interno, va a aumentar la calidad óptica del pozo foveal al disminuir la expansión de la luz8

Se cree que la zona foveal avascular (FAZ) debería ser el foco principal en el análisis de la estructura vascular macular para demostrar la patogénesis del glaucoma. La degeneración o los cambios atróficos de los capilares maculares pueden afectar la forma y el tamaño de la FAZ9

El principio de interferometría de Michelson (Premio Nobel 1911) fue el impulsador de la visualización de la mácula, según un cohorte tomográfico en la que se diferencia el resto de capas de la retina y el epitelio pigmentario de la retina10. En 2006, se perfecciona los primeros angiogramas por OCT y en el 2011 se desarrolla OCT-A .La principal beneficio de la OCT-A es que no es invasivo, tampoco usa contraste o hay efectos adversos 11.

La OCT- A tiene la competencia de instaurar imágenes por la detección de desplazamiento de la sangre en los vasos sanguíneos, esto se consigue por la suma de múltiples A- scan que constituyen imágenes $B$ - scan de la retina, también de la colocación de numerosos B- scan obtenidos sucesivamente en la misma ubicación se genera la imagen volumétrica de la OCT-A12.

OCT-A en el glaucoma, es útil para el diagnóstico y control de pacientes con glaucoma. Ya que en la fisiopatología existe otra teoría que es la vascular, se apoya en que se encuentra alteraciones de base en el flujo sanguíneo de la cabeza del disco óptico13. Entonces se puede localizar la densidad media de los capilares en el disco, así como en el área peripapilar y macular en estos pacientes en comparación con los pacientes sanos 14 .

Según estudios no se han encontrado patrones de diámetros exactos sobre el área foveolar, pero si se han hallado que existe un aumento de la zona foveal avascular en pacientes con glaucoma, por ello se tratará de identificar, conforme a las pruebas se permitirá valorar si la Presión intraocular no controlada también hace que aumente este aumento de la FAZ15

La OCT-A tendría que ser fundamental para el diagnóstico de pacientes con Glaucoma normotensional, se evidencia que la zona foveal avascular es más larga y hay una disminución de la densidad de vasos maculares 16 .

El perímetro de la zona foveal avascular tuvo una buena capacidad de diagnóstico para poder diferenciar los ojos glaucomatosos con defecto visual en campo central de ojos sanos13. El índice de circularidad sería un biomarcador que representa la interrupción de la red capilar parafoveal en el glaucoma17. Se espera, establecer si existen cambios del tamaño de la zona foveal avascular en pacientes con glaucoma a comparación de pacientes sanos.

\section{MATERIALES Y MÉTODOS}

El presente estudio es una Revisión Sistemática y Metaanálisis. La Población de estudio fueron los artículos originales que cumplan los criterios de inclusión y exclusión en los que se relacionen las variables de cambio en el tamaño de la zona foveal avascular en pacientes con glaucoma y pacientes sanos.

Criterios de inclusión: artículos originales, Antigüedad no mayor a 5 años, El factor de estudio debe estar presente en los artículos, Artículos con población adulta (mayores de 18 años), Artículos retrospectivos, Artículo con un tamaño de muestra mínimo de 40 personas, La variable respuesta debe estar presente en los artículos, Artículos en español, inglés, francés, alemán, Artículos que incluyan como evaluación la Angio- OCT. Criterios de exclusión: Estudios reporte de casos, serie de casos, Estudios en población obstétrica, Estudios en población pediátrica El tipo de muestreo es Muestreo no probabilístico. La unidad de muestreo son los artículos originales.

\section{Aspectos éticos:}

La investigación se realizó con el debido respeto al lineamiento ético y moral, que está redactado en las líneas de investigación biomédica, conforme a la ley general de salud $\left(\mathrm{N}^{\circ} 26842\right)$ y el Código de Ética y deontología del colegio médico del Perú.

Además, la investigación respetó la normativa internacional y nacional que regula la investigación con seres humanos, como la declaración de Helsinki. El artículo 46 de la ley general de salud, de la información obtenida de la investigación, será presentada sin incurrir en el plagio y falsificación.

\section{RESULTADOS}

Se procedió a la búsqueda de artículos científicos de acuerdo a criterios de inclusión y exclusión según plateados en los diferentes buscadores como PUBMED, OVID, Cochrane Library, Portal Regional de la BVS, Science Direct y Clinical Key.

Se encontró un total de 272 artículos de los cuales 26 en PUBMED, 2 en OVID, 0 en Cochrane Library 26 en BVS, 155 en Science Direct y por último 63 en Clinical Key. Se tuvo que proceder a la eliminación de 72 artículos por estar duplicados, quedando 200 artículos que se procedió a ser evaluados según título y resumen. Luego se descartó 7 artículos por ser en 
población pediátrica, 175 no incluía a la variable glaucoma, 8 no relacionaba la variable zona foveal avascular, 3 asociaron las dos variables de manera que no nos es útil en esta revisión. Seguidamente luego de la eliminación, de los artículos fueron evaluados en texto completo para su elegibilidad. Se halló que 2 de los artículos no contaban con los datos completos. En final, se selecciona 5 artículos para el análisis cualitativo y cuantitativo. Los pacientes participantes de los cinco artículos seleccionados sumaron un total de 574 siendo 353 pacientes con diagnóstico de glaucoma y 221 pacientes sanos. Se analizó la variable del área de zona foveal avascular de los tres estudios incluidos, variables continua la diferencia de medias (MD) y diferencia de medias estandarizadas. Los artículos fueron de estudios observacionales, retrospectivos y transversales en los cuales se compara el tamaño de zona foveal avascular en pacientes con glaucoma y pacientes sanos. Todo este proceso es según diagrama PRISMA (Diagrama $N^{\circ} 1$ ).

Diagrama N¹: Flujo PRISMA de la selección de artículos que comparen los cambios en el tamaño de la zona foveal avascular en pacientes con diagnóstico de glaucoma y en pacientes sanos.

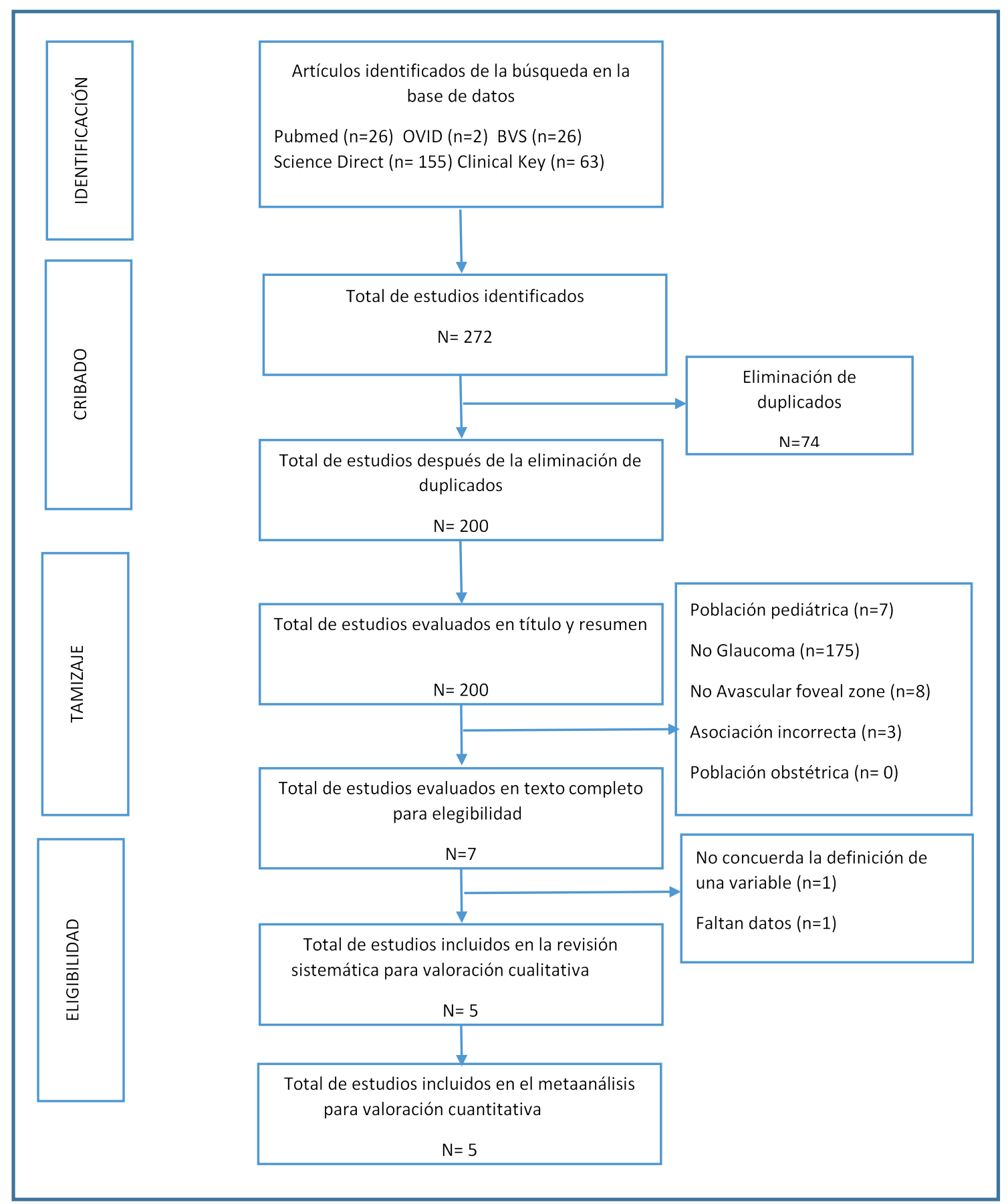

\section{Sesgo:}

Se utilizó NEWCASTLE - OTTAWA QUALITY ASSESSMENT SCALE (adapted for cross sectional studies) que es adaptado para estudios transversales que evalua selección, comparación y resultado. Se utilizó el software de Review Manager 5.4.1. En los Diagramas №2 y Diagrama $\mathrm{N}^{\circ} 3$ se muestra los resultados. 
Revisión sistemática: Cambios en el tamaño de la zona foveal avascular en pacientes con diagnóstico de glaucoma y en pacientes sanos.

Diagrama $\mathbf{N}^{\circ}$ 2: Sesgo en porcentaje de los estudios incluidos.

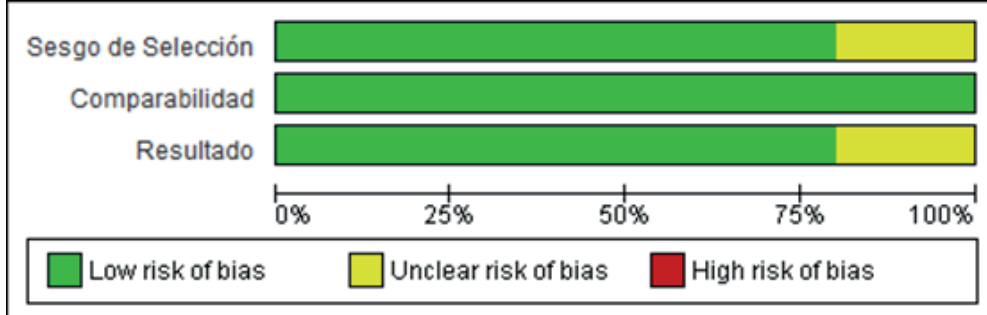

Diagrama $\mathbf{N}^{\circ}$ 3: Sesgo de los estudios incluidos.

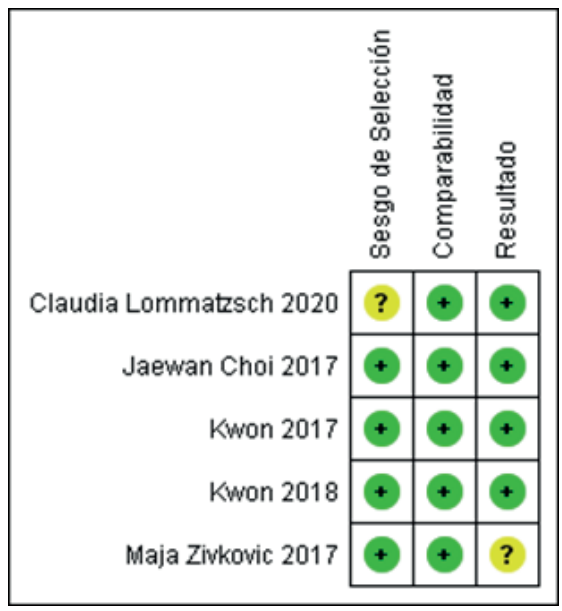

\section{Variables:}

Se analizó la variable del área de zona foveal avascular de los tres estudios incluidos, variables continua la diferencia de medias (MD) y diferencia de medias estandarizadas. Se usó el software de Review Manager 5.4.1 para evaluarlo.

\section{DISCUSIÓN}

Existe evidencia de que la morfología del área foveal avascular está relacionada con muchas afecciones patológicas del globo ocular; se ha demostrado que su tamaño y regularidad son tanto de diagnóstico y valor pronóstico en enfermedades retinianas tales como retinopatía diabética y oclusión de las venas retinianas. En el glaucoma, las alteraciones microcirculatorias en la perifovea se correlacionan espacialmente con la pérdida de agudeza visual central. La pérdida de circularidad del área foveal vascular se asocia significativamente con la presencia de defecto de agudeza visual central, suponiendo que la pérdida focal de la red capilar parafoveal puede alterar la forma y el tamaño del área foveal avascular en el glaucoma18,19.

En el presente metanalisis se pudo observar que existen pocas investigaciones que evalúen la variación del tamaño del área foveal avascular entre pacientes con o sin glaucoma; las 5 publicaciones encontradas corresponden todas a estudios transversales, con tamaño muestrales discretos; en todos los casos son publicaciones recientes, de los últimos 5 años y al realizar la valoración del riesgo de sesgo; solo 2 de ellas presentan un riesgo indeterminado de sesgo, uno para el criterio de selección y otro para el criterio de resultados.

El estudio de Lommatzsch C. en Alemania del año 2020 evaluaron además de la zona avascular foveal, al perímetro y índice de acircularidad y densidad de vasos circundantes, en ojos glaucomatosos en comparación con ojos sanos, con subdivisión adicional en defectos del campo visual en diferentes ubicaciones en 128 ojos; concluyendo que en el glaucoma, el área avascular foveal se agranda cuando el defecto del campo visual está centralizado y la densidad vascular disminuye significativamente en el área de la fóvea20. El estudio de Kwon J, en China en el 2018; evalúan la relación entre el tamaño de la zona avascular foveal tanto en su área como en el perímetro, con la densidad vascular en varias regiones de la retina usando angiografía por tomografía de coherencia óptica, en pacientes con glaucoma de ángulo abierto y un grupo de control de ojos normales; concluyendo que el tamaño del área avascular foveal puede ser un biomarcador potencial que representa el estado de células ganglionares retinianas y de la función visual central en pacientes con glaucoma18.

El estudio de Zivkovic M, en Eslovenia en el 2017; se ocuparon de medir el diámetro de la zona avascular foveal, el área y la densidad de los vasos mediante angiografía por tomografía de coherencia óptica en pacientes con glaucoma de tensión normal y otro grupo de sujetos sanos, encontrando que múltiples parámetros de esta área fueron estadísticamente significativamente aumentados en el grupo con glaucoma en comparación con los del grupo de control, siendo el primer estudio que arrojará luz en investigación adicional sobre la asociación entre estas 2 variables; reconocen que podría tener un papel importante en el futuro en diagnóstico de pacientes con glaucoma normotensivo así como en la comprensión de su fisiopatología19.

El estudio de Choi J. en China en el 2017, evaluó las características cuantitativas de la densidad de los vasos maculares y la zona avascular foveal en el glaucoma mediante angiografía por tomografía de coherencia óptica respecto a ojos de 52 participantes sanos; encontraron además que hubo un aumento del perímetro y una disminución del índice de circularidad del área avascular foveal en el glaucoma en comparación con los controles21.

Finalmente el estudio de Kwon J. en China en el 2017, evaluaron la capacidad diagnóstica de los parámetros de la zona avascular foveal para discriminar ojos glaucomatosos con defectos del campo visual en diferentes ubicaciones (central versus periférico) de los ojos normales; identificando al área avascular foveal, su perímetro y su circularidad fueron mejores para diferenciar los ojos glaucomatosos de los ojos normales reconociéndolos como potenciales biomarcadores de diagnóstico potencial para detectar pacientes glaucomatosos22.

Además de la asociación evaluada, existe en los estudios como denominador común la hipótesis de si el perímetro y el índice de circularidad, pueden reflejar también el estado de perfusión vascular macular como pérdida focal de la arcada capilar parafoveal que incluso tiende a ocurrir antes del agrandamiento del área foveal avascular en la enfermedad avanzada. En este sentido la precisión diagnóstica relativamente baja de la densidad vascular macular fuera de la región foveal puede implicar que la pérdida focal de capilares en la etapa temprana del glaucoma no se refleja bien en este parámetro sectorial fuera de la fóvea calculada a partir de imágenes tomográficas, en contraste, el cambio sutil de la arcada vascular parafoveal puede hacer que el contorno del área avascular foveal sea más irregular, resultando en la disminución del índice de circularidad 
en la etapa temprana de la enfermedad, ya que solo hay un capa única de soporte vascular en esta área. Por ello el análisis de estos parámetros vasculares cobran importancia progresiva en términos de diagnóstico y pronostico en pacientes con glaucoma21,22.

Se concluye, en la revisión sistemática realizada se encontró que existen diferencias significativas respecto al tamaño de la zona foveal avascular entre pacientes con diagnóstico de glaucoma o pacientes sanos. Se sugiere, que los hallazgos descritos debieran ser considerados como sustento al momento de diseñar estrategias orientadas a mejorar la capacidad diagnostica del glaucoma así como el pronóstico de su evolución para orientar las estrategias terapéuticas.

Diagrama $\mathbf{N}^{\circ} \mathbf{4}$ : Pacientes con glaucoma vs pacientes sanos, cambios en el área foveal avascular.

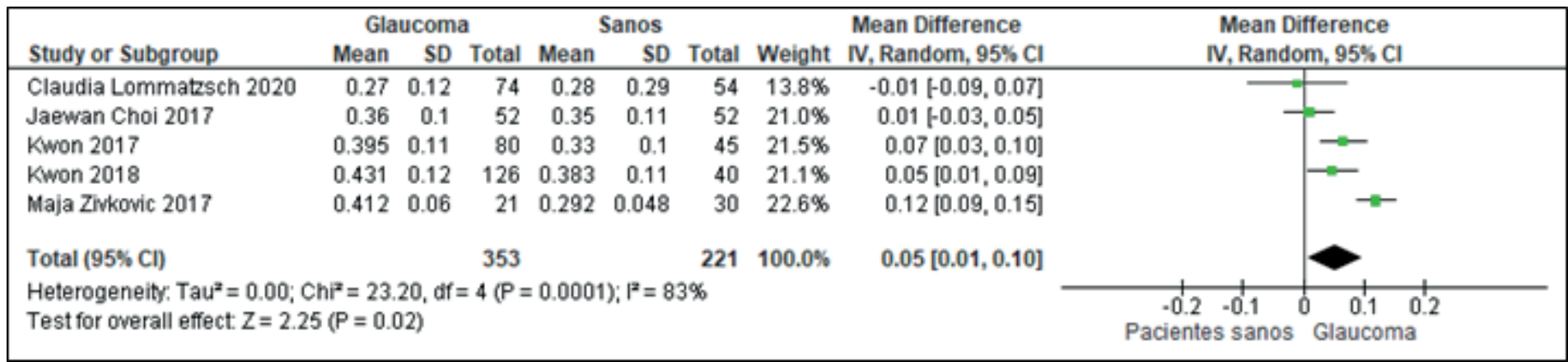

Diagrama $\mathbf{N}^{\circ}$ 5: Pacientes con glaucoma con defecto visual central vs pacientes con glaucoma con defecto visual periférico, cambios en su área foveal avascular

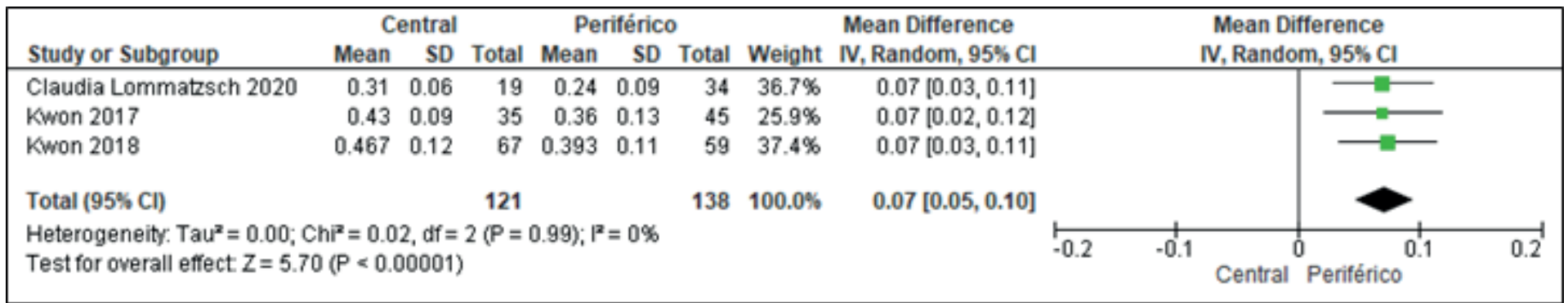




\section{REFERENCIAS BIBLIOGRÁFICAS}

1. Quigley H.A. and A.T. Broman, The number of people with glaucoma worldwide in 2010 and 2020. Br J Ophthalmol, 2006. 90(3): p. 262-7.

2. Križaj D. What is glaucoma?. 2019 May 30. In: Kolb $\mathrm{H}_{\text {, }}$ Fernandez E, Nelson R, editors. Webvision: The Organization of the Retina and Visual System. Salt Lake City (UT): University of Utah Health Sciences Center.

3. $\mathrm{Xu} \mathrm{L}$, Wang $\mathrm{X}, \mathrm{Wu}$ M. Topical medication instillation techniques for glaucoma. Cochrane Database Syst Rev. 2017;(2):CD010520. PubMed PMID: 28218404

4. Jonas JB, Aung T, Bourne RR, Bron AM, Ritch R, Panda-Jonas S. Glaucoma. Lancet. 2017;390(10108):2183-93.

5. Liu B, McNally S, Kilpatrick JI, Jarvis SP, O'Brien CJ. Aging and ocular tissue stiffness in glaucoma. Surv Ophthalmol. 2018;63(1):56-74.

6. Shahlaee, A., Pefkianaki, M., Hsu, J., \& Ho, A. C. Measurement of Foveal Avascular Zone Dimensions and its Reliability in Healthy Eyes Using Optical Coherence Tomography Angiography. American Journal of Ophthalmology, (2016) 161, 50-55.e1.

7. Balaratnasingam, C., Inoue, M., Ahn, S., McCann, J., Dhrami-Gavazi, E., Yannuzzi, L. A., \& Freund, K. B. Visual Acuity Is Correlated with the Area of the Foveal Avascular Zone in Diabetic Retinopathy and Retinal Vein Occlusion. Ophthalmology, (2016). 123(11), 2352-2367.

8. Chui, T. Y. P., Zhong, Z., Song, H., \& Burns, S. A. Foveal Avascular Zone and Its Relationship to Foveal Pit Shape. Optometry and Vision Science, (2012). 89(5), 602-610.

9. Mammo, Z., Balaratnasingam, C., Yu, P., et al., Quantitative noninvasive angiography of the fovea centralis using speckle variance optical coherence tomography. 2015

10. Dr. Carlos Orduna, Angio OCT, la nueva tecnica para el diagnostco oftalologico, 15 dic 2018, Disponible en https://drusasmaculares.com/angio-oct-la-nueva-tecnica-p ara-el-diagnostico-oftalmologico/\#faqs

11. de Carlo TE, Romano A, Waheed NK, Duker JS. A review of optical coherence tomography angiography (OCTA). Int J Retin Vitr. 2015;1(1):5

12. Chalam K, Sambhav K. Optical coherence tomography Angiography in retinal diseases. J Ophthalmic Vis Res. 2016;11(1):84-92

13. Wylęgała A, Teper S, Dobrowolski D, Wylęgała E. Optical coherence angiography:A review. Medicine. 2016;95(41):e4907

14. 14. Mammo Z, Heisler M, Balaratnasingam C, Lee $S$, Yu DY, Mackenzie $P$, et al. Quantitative Optical Coherence Tomography Angiography of Radial Peripapillary Capillaries in Glaucoma, Glaucoma Suspect, and Normal Eyes. Am J Ophthalmol. 2016; 170:41-9.

15. Choi W, Moult E. M, Waheed N. K, Adhi M, Lee B, Lu C. D, de Carlo T. E, Jayaraman V, Rosenfeld P. J, Duker J. S, and Fujimoto J. G. Ultrahigh-Speed, Swept-Source Optical Coherence Tomography Angiography in Nonexudative AgeRelated Macular Degeneration with Geographic Atrophy. Ophthalmology. 2015 122(12):2532-44

16. Mesa, E. V., Goyeneche, H. F. G., \& Malo, L. C. M. "Angiografía por tomografía de coherencia óptica: una nueva herramienta diagnóstica". Revista Sociedad Colombiana de Oftalmología, (2018) Vol 51(1), 63-71.

17. W. A. Samara, E. A. T. Say, C. T. L. Khoo et al., "Correlation of foveal avascular zone size with foveal morphology in normal eyes using optical coherence tomography angiography," Retina, vol. 35, no. 11, pp. 2188-2195, 2015.

18. Kwon, J., Choi, J., Shin, J. W., Lee, J., \& Kook, M. S. Alterations of the foveal avascular zone measured by optical coherence tomography angiography in glaucoma patients with central visual field defects. Investigative ophthalmology \& visual science, (2017). 58(3), 1637-1645.

19. Zivkovic, M., Dayanir, V., Kocaturk, T., Zlatanovic, M., Zlatanovic, G., Jaksic, V. \& Jovanovic, S. Foveal avascular zone in normal tension glaucoma measured by optical coherence tomography angiography. BioMed research international, 2017. 7 pages

20. Lommatzsch C. Does the Foveal Avascular Zone Change in Glaucoma?] Klin Monbl Augenheilkd . 2020 Jul;237(7):879-888.

21. Choi J. Quantitative optical coherence tomography angiography of macular vascular structure and foveal avascular zone in glaucoma PLoS ONE 2017U; 12(9):e0184948.

22. Kwon J. Glaucoma Diagnostic Capabilities of Foveal Avascular Zone Parameters Using Optical Coherence Tomography Angiography According to Visual Field Defect Location Journal of Glaucoma 2017; 26(12):1. 